\title{
Type 1 Kounis Syndrome Triggered by Noradrenaline in a case of Pyogenic Meningitis
}

JeR

Ramya SG, Jerryn Raj Selvyn, Abdur Rahman S, Nellaiappa Ganesan SK, Sundaravadivelu V Department of General Medicine, SRM Medical College, Kattankulathur, Tamil Nadu, India.

Abstract:

Kounis syndrome is the coincidental occurrence of acute coronary syndromes with allergic or hypersensitivity reactions. Kounis syndrome has been established as a hypersensitivity coronary disorder induced by various conditions, drugs, environmental exposures, food items and drug eluting coronary stents. It is not a rare disease but an infrequently diagnosed clinical entity. Clinicians should be aware that various mediators of allergy can cause coronary spasm and even plaque rupture and thrombus formation, thereby causing a serious impact on the course, prognosis and management of the allergic reaction. Here we report a rare case of Type 1 Kounis syndrome triggered by nordrenaline in a patient with pyogenic meningitis.

Key words: Acute Coronary Syndrome, Angina Pectoris, Coronary Disease, Hypersensivity, Spasm.

\section{Introduction}

In 1991, Kounis and Zavras described the coincidental occurrence of acute coronary syndromes with allergic reactions. Since then allergic angina and allergic acute myocardial infarction have been recognized as Kounis syndrome [1]. This has been reported in association with a variety of drugs, insect sting, food, environmental exposures and medical conditions. In this article, we describe a rare case of type 1 Kounis syndrome caused by noradrenaline which was initiated for septic shock.

\section{Case Report}

A 24 years old male working as cook was admitted with complaints of fever, vomiting and headache since 4 days and altered behaviour with irritability since two days. He had no significant past or family history and no history of any drug abuse. On examination he was drowsy and he did not follow any commands but he was responding to painful stimuli by opening eyes and withdrawal of limbs when tested on each of them. There was no evidence of any focal neurological deficit. Kernig's sign and Brudzinki's sign were positive and plantar reflex was equivocal bilaterally.

On investigating, complete hemogram showed mildly raised leucocyte count $(13,000$ cells $/$ cu.mm) with neutrophilia and ESR was $45 \mathrm{~mm} / \mathrm{hr}$. His chest X-ray, liver function tests and renal function tests were normal. CT brain was normal. Cerebrospinal fluid analysis showed 850 cells of

\section{Corresponding Author: Dr. Ramya SG}

Email: dr.sgramya@gmail.com

Received: April 7, 2016 | Accepted: June 23, 2016 | Published Online: August 25, 2016

This is an Open Access article distributed under the terms of the Creative Commons Attribution License (creativecommons.org/licenses/by/3.0)

Conflict of interest: None declared | Source of funding: Nil | DOl: http://dx.doi.org/10.17659/01.2016.0097 
which $80 \%$ were polymorphs with high protein and low sugar suggestive of pyogenic meningitis for which appropriate antibiotics were started. He developed septic shock after four hours of admission and mechanical ventilation started in view of poor consciousness. Resuscitation with fluids was started initially with which BP did not improve and noradrenaline drip was initiated. He developed profuse sweating, tachycardia and worsening hypotension immediately. Electrocardiogram showed ST segment elevation in leads I, II, aVL, $\mathrm{aVf}, \mathrm{V} 4, \mathrm{~V} 5$ and $\mathrm{V} 6$ as shown in figure 1. Troponin $T$ was elevated. Noradenaline drip was stopped immediately. Adrenaline and hydrocortisone was given with fluid resuscitation. ST segment elevation which was previously noted in electrocardiogram returned to baseline within ten minutes as shown in figure 2 and his vital parameters improved. Echocardiogram was normal. Coronary angiogram was performed before discharge and it did not show any abnormality. Patient was counselled and informed regarding the coronary vasospasm that occurred following noradrenaline injection.

\section{Discussion}

Kounis syndrome is a group of symptoms that manifests as unstable angina, or acute myocardial infarction and is triggered by the release of inflammatory mediators following an allergic insult. It is caused by inflammatory mediators such as histamine, neutral proteases, arachidonic acid products, platelet activating factor and a variety of cytokines and chemokines released during the activation process [2]. Allergens such as foods, insect venom, iodine contrast media or drugs induce mast cell degranulation, resulting in the release of a number of vasoactive mediators (histamine, leukotrienes, serotonin) and proteases (tryptase, chymase) both locally and into the systemic bloodstream [3]. The exact pathophysiology of Kounis syndrome is still not clear [4].

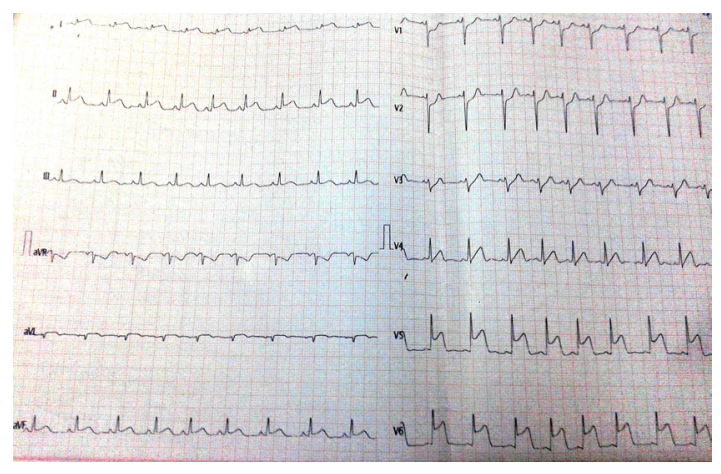

Fig. 1: ST elevation in leads I, II, avL, avF, V4, V5, V6 following initiation of noradrenaline.

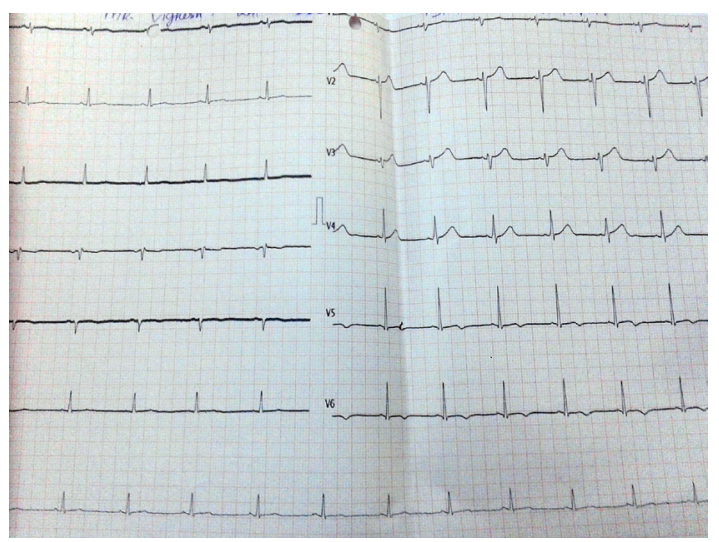

Fig.2: Resolution of ECG changes after stopping noradrenaline.

There are three variants of Kounis syndrome. Type I variant includes patients with normal or nearly normal coronary arteries without predisposing factors for coronary artery disease. Type II variant includes patients with culprit but quiescent pre-existing atheromatous disease in whom the acute allergic attacks can induce either coronary artery spasm, or plaque erosion or rupture manifesting as acute myocardial infarction. Hypersensitivity reactions following implantation of drug-eluting stents and stent thrombosis which may be considered as a manifestation of Kounis syndrome, a type III variant of Kounis syndrome has been introduced $[5,6]$. 
Drugs implicated with Kounis syndrome include catecholamines (epinephrine, norepinephrine, isoproterenol, dopamine, dobutamine); parasympathomimetic agents (acetylcholine, methacholine, pilocarpine); anticholinesterase agents (neostigmine); beta-blockers; anaesthetic agents (etomidate); antibiotics (ciprofloxacin, amoxicillin, ampicillin, cephalosporins, vancomycin); NSAIDS; chemotherapeutic drugs; thrombolytic agents; withdrawal from chronic exposure to nitroglycerine, cocaine, smoking and alcohol [7-10]. No biomarker accurately predicts the risk of Kounis syndrome, albeit recent reports link elevated serum tryptase levels to increased susceptibility to allergic reactions as well as asymptomatic ACS. Most recommendations are based on anecdotal case reports or case series [1 11 .

The management of Kounis syndrome may be challenging for clinicians as the international consensus are still unavailable. Patients with Kounis syndrome need treatment with steroids, antihistamines, fluid resuscitation, oxygen and vasodilators. The treatment should both dilate coronary vessels and suppress the allergic reaction. Vasodilator drugs, including nitrates and calcium channel blockers should be considered as first-line therapy in young and previously healthy individuals, since vasospasm is the primary mechanism.

\section{Conclusion}

Though epinephrine and dobutamine [12] are reported to be associated with Kounis syndrome, noradrenaline has not been reported so far to cause the same and hence our case report is first to report the association of Kounis syndrome with noradrenaline. Kounis syndrome was identified before two decades, but the pathophysiology and the guidelines for management are still lacking. Hence further studies are recommended to characterize the epidemiology, clinical features, diagnosis and to define appropriate preventive and interventional measures as it may be fatal.

\section{References}

1. Kounis NG, Zavras GM. Allergic angina and allergic myocardial infarction. Circulation. 1996;94(7):1789.

2. Kounis NG. Kounis syndrome (allergic angina and allergic myocardial infarction): Anatural paradigm? Int J Cardiology. 2006;1 10:7-14.

3. Mazarakis A, Koutsojannis CM, Kounis NG, Alexopoulos D. Cefuroxime-induced coronary artery spasm manifesting as Kounis syndrome. Acta Cardiol. 2005;60:341-345.

4. Zavras GM, Papadaki PJ, Kokkinis CE, Kalokairinov $K$, Kouni SN, Batsolaki $M$, et al. Kounis syndrome secondary to allergic reaction following shellfish ingestion. Int J Clin Pract. 2003;57:622-624.

5. Nikolaidis LA, Kounis NG, Grandman AH. Allergic angina and allergic myocardial infarction: a new twist on an old syndrome. Can J Cardiol. 2002; 18:508-511.

6. Biteker M. A new classification of Kounis syndrome. Int J Cardiol. 2010;145:553.

7. Kounis NG, Soufras GD, Lianas D, Patsouras N. After Administration of Intravenous Epinephrine for bee Sting-induced Anaphylaxis: Kounis Syndrome or Epinephrine Effect? Chin Med J. 2016;1 29:500-501.

8. Horak A, Raine R, Opie LH, Lloyd EA. Severe myocardial ischaemia induced by intravenous adrenaline. BMJ. 1983;1:519.

9. Ridella M, Bagdure S, Nugent K, Cevik C. Kounis syndrome following betalactam antibiotic use: review of literature. Inflamm Allergy Drug Targets. 2009;8:11-16.

10. De Groot JW, Gosselink AT, Ottervanger JP. Acute ST segment elevation myocardial infarction associated with diclofenac induced anaphylaxis: case report. Am J Crit Care. 2009; 18:386-388.

11. Van Ginkel A, Sorgdrager B, de Graaf MA, Karalis I, Ajmone Marsan N. ST-segment elevation associated with allergic reaction to echocardiographic contrast agent administration. Netherlands Heart Journal. 2014;22(2):77-79.

12. Cevik C, Nugent K, Shome GP, Kounis NG. Treatment of Kounis syndrome. Int J Cardiol. 2010;143:223-226. 